\title{
Video Article \\ Catheter Ablation in Combination With Left Atrial Appendage Closure for Atrial Fibrillation
}

\author{
Martin J. Swaans ${ }^{1}$, Arash Alipour ${ }^{1}$, Benno J.W.M. Rensing ${ }^{1}$, Martijn C. Post ${ }^{1}$, Lucas V.A. Boersma \\ ${ }^{1}$ Department of Cardiology, St. Antonius Hospital, The Netherlands
}

Correspondence to: Martin J. Swaans at m.swaans@antoniusziekenhuis.nl

URL: https://www.jove.com/video/3818

DOI: doi: $10.3791 / 3818$

Keywords: Medicine, Issue 72, Anatomy, Physiology, Biomedical Engineering, Immunology, Cardiology, Surgery, catheter ablation, WATCHMAN, LAA occlusion, atrial fibrillation, left atrial appendage, warfarin, oral anticoagulation alternatives, catheterization, ischemia, stroke, heart, vein, clinical, surgical device, surgical techniques, Vitamin $\mathrm{K}$ antagonist

Date Published: 2/26/2013

Citation: Swaans, M.J., Alipour, A., Rensing, B.J., Post, M.C., Boersma, L.V. Catheter Ablation in Combination With Left Atrial Appendage Closure for Atrial Fibrillation. J. Vis. Exp. (72), e3818, doi:10.3791/3818 (2013).

\section{Abstract}

Atrial fibrillation (AF) is the most common sustained cardiac arrhythmia, affecting millions of individuals worldwide ${ }^{1-3}$. The rapid, irregular, and disordered electrical activity in the atria gives rise to palpitations, fatigue, dyspnea, chest pain and dizziness with or without syncope ${ }^{4,5}$. Patients with $\mathrm{AF}$ have a five-fold higher risk of stroke ${ }^{6}$.

Oral anticoagulation (OAC) with warfarin is commonly used for stroke prevention in patients with AF and has been shown to reduce the risk of stroke by $64 \%{ }^{7}$. Warfarin therapy has several major disadvantages, however, including bleeding, non-tolerance, interactions with other medications and foods, non-compliance and a narrow therapeutic range ${ }^{8-11}$. These issues, together with poor appreciation of the risk-benefit ratio, unawareness of guidelines, or absence of an OAC monitoring outpatient clinic may explain why only $30-60 \%$ of patients with $\mathrm{AF}$ are prescribed this drug ${ }^{8}$.

The problems associated with warfarin, combined with the limited efficacy and/or serious side effects associated with other medications used for $A F^{12,13}$, highlight the need for effective non-pharmacological approaches to treatment. One such approach is catheter ablation (CA), a procedure in which a radiofrequency electrical current is applied to regions of the heart to create small ablation lesions that electrically isolate potential AF triggers ${ }^{4}$. CA is a well-established treatment for AF symptoms ${ }^{14,15}$, that may also decrease the risk of stroke. Recent data showed a significant decrease in the relative risk of stroke and transient ischemic attack events among patients who underwent ablation compared with those undergoing antiarrhythmic drug therapy ${ }^{16}$

Since the left atrial appendage (LAA) is the source of thrombi in more than $90 \%$ of patients with non-valvular atrial fibrillation ${ }^{17}$, another approach to stroke prevention is to physically block clots from exiting the LAA. One method for occluding the LAA is via percutaneous placement of the WATCHMAN LAA closure device. The WATCHMAN device resembles a small parachute. It consists of a nitinol frame covered by fabric polyethyl terephthalate that prevents emboli, but not blood, from exiting during the healing process. Fixation anchors around the perimeter secure the device in the LAA (Figure 1). To date, the WATCHMAN is the only implanted percutaneous device for which a randomized clinical trial has been reported. In this study, implantation of the WATCHMAN was found to be at least as effective as warfarin in preventing stroke (allcauses) and death (all-causes) ${ }^{18}$. This device received the Conformité Européenne (CE) mark for use in the European Union for warfarin eligible patients and in those who have a contraindication to anticoagulation therapy ${ }^{19}$.

Given the proven effectiveness of CA to alleviate AF symptoms and the promising data with regard to reduction of thromboembolic events with both CA and WATCHMAN implantation, combining the two procedures is hoped to further reduce the incidence of stroke in high-risk patients while simultaneously relieving symptoms. The combined procedure may eventually enable patients to undergo implantation of the WATCHMAN device without subsequent warfarin treatment, since the CA procedure itself reduces thromboembolic events. This would present an avenue of treatment previously unavailable to patients ineligible for warfarin treatment because of recurrent bleeding ${ }^{20}$ or other warfarin-associated problems.

The combined procedure is performed under general anesthesia with biplane fluoroscopy and TEE guidance. Catheter ablation is followed by implantation of the WATCHMAN LAA closure device. Data from a non-randomized trial with 10 patients demonstrates that this procedure can be safely performed in patients with a CHADS 2 score of greater than $1^{21}$. Further studies to examine the effectiveness of the combined procedure in reducing symptoms from $\mathrm{AF}$ and associated stroke are therefore warranted.

\section{Video Link}

The video component of this article can be found at https://www.jove.com/video/3818/ 


\section{Pre-procedure Preparation}

1. Prior to performing the procedure, discuss the risks and benefits of the procedure with the patient, and obtain a signed consent form. Adjust the patient's vitamin $\mathrm{K}$ antagonist dose to achieve an international normalized ratio of 2.0 to 3.0. Continue antiarrhythmic drug therapy up to the time of the procedure.

2. Either the day before or the day of the procedure, perform a transesophageal echocardiogram (TEE) to document the absence of thrombi within the LAA, assess the features and type of the LAA, and to determine the appropriate WATCHMAN device size to implant (see the 2012 JoVE article by Mobius-Winkler and colleagues for more information) ${ }^{22}$.

3. Perform the combined catheter ablation and WATCHMAN LAA Closure Device placement procedure in an electrophysiology lab with the appropriate diagnostic and imaging equipment for the procedure.

4. With the patient in the supine position, administer anaesthesia according to institutional protocols.

\section{Catheter Ablation}

1. Ensure that the electrophysiological recording system (Bard Inc., Lowell, MA) is using filter settings of $100 \mathrm{ko} 500 \mathrm{kHz}$ and a signal amplification of 5,000 .

2. Once the patient is fully anesthetized, administer $20 \mathrm{ml}$ of $1 \%$ lidocaine as a local anesthetic in the groin region. Immediately, clean and prep the inguinal region for cannulation.

3. Locate the femoral vein, and insert a venous 8 French $(8 \mathrm{~F})$ sheath. Introduce a quadripolar catheter into the coronary sinus for pacing purposes. Then, insert a long guidewire, and remove the sheath. Introduce a standard venous transseptal access sheath.

4. Remove the long guidewire, and insert a Transseptal RF Brockenbrough needle (Baylis Medical Company Inc., Montreal, Quebec, Canada) with a 12.5F-outer-diameter/9.5F-inner-diameter steerable sheath (Channel, Bard, Lowell, MA) and perform a transseptal puncture.

5. After crossing the interatrial septum, position a pigtail catheter in the center of the left atrium and perform a 3D rotational angiography.

6. The Pulmonary Vein Ablation Catheter (PVAC: Medtronic/Ablation Frontiers, Inc., Carlsbad, CA) is a 9F, over-the-wire, circular, decapolar mapping and ablation catheter with a 25-mm-diameter array at the distal tip. No additional nonfluoroscopic guiding or steering systems were used. The PVAC has ten electrodes on a nitinol frame that enable mapping, ablation and verification of pulmonary vein isolation. Insert a 0.032-inch guidewire into the PVAC.

7. Next, insert the whole system into the venous sheath. The information gathered by the PVAC is displayed on a monitor to generate a 3D image of the patient's heart with the catheter's location displayed.

8. Deliver a bolus of 10.000 IE heparin to prevent clotting as a result of the procedure. Position the wire in one of the branches of the pulmonary vein, then advance the PVAC into the pulmonary vein antrum.

9. Use fluoroscopic guidance, electrograms, and $3 \mathrm{D}$ electric signal imaging (ESI) reconstruction to assess the position of the PVAC and adjust it to get the best contact with all of the electrodes.

10. Once a satisfying catheter position is achieved, commence radio frequency (RF)-ablation by selecting the appropriate electrode pairs for delivery of RF energy.

11. Set the generator to adjust the power to achieve a target temperature of $60^{\circ} \mathrm{C}$ for each electrode in the selected pairs. In most cases, all electrodes pairs will be activated during the first applications to create overlapping lesion rings.

12. Ablate for $60 \mathrm{sec}$. Check the monitor for conditions during ablation. If the temperature does not rise above $50{ }^{\circ} \mathrm{C}$ within $15 \mathrm{sec}$, discontinue the application, adjust the position of the electrodes and start again.

13. Move and rotate the PVAC-catheter to create circumferential pulmonary vein lesions.

14. Repeat this procedure for all pulmonary veins.

15. After creating circumferential lesions for all pulmonary veins, restore sinus rhythm by direct current cardioversion (DCCV).

16. Confirm isolation by checking the absence of local potentials (entrance and exit block) with PVAC mapping inside each vein combined with pacing maneuvers from the coronary sinus.

17. For residual potentials on isolated electrode pairs, target ablation only on that and adjacent pairs to facilitate reaching target temperature.

\section{Implantation of the WATCHMAN Device}

1. Once the catheter ablation procedure is complete, the WATCHMAN device is implanted.

2. Advance a 0.035 " stiff guidewire (e.g. Amplatz Super stiff $260 \mathrm{~cm}$ ) into the PVAC and position the guidewire in the left upper pulmonary vein. Remove the PVAC while maintaining the position of the guidewire.

3. If ablation was not performed prior to implantation, administer heparin at $100 \mathrm{lU} / \mathrm{kg}$ bodyweight to obtain an activated clotting time of $200-300$ $\mathrm{sec}$. Check the activated clotting time level to ensure that it is still $200-250 \mathrm{sec}$. Continue to monitor this every 30 min as needed.

4. Remove the WATCHMAN Access System and dilator from the packaging under sterile conditions. Carefully inspect it for damage.

5. Fill a 60CC syringe with saline and flush the side port of the WATCHMAN access sheath. Use the remaining saline to flush the dilator. Insert the dilator into the WATCHMAN access sheath.

6. Advance the WATCHMAN access sheath and dilator over the guidewire into the left atrium. As the WATCHMAN access sheath nears the center of the left atrium, hold the dilator and advance the WATCHMAN access sheath to the initial position of the left atrium only.

7. Remove the dilator and guidewire. Before tightening the hemostasis valve, allow back bleed to minimize the potential for introducing air. Flush WATCHMAN access sheath with saline.

8. Tighten the valve on the access sheath.

9. To confirm the LAA dimensions, use TEE to measure the maximum LAA dimensions in 4 views at $0^{\circ}, 45^{\circ}, 90^{\circ}$ and $135^{\circ}$.

10. Flush the pigtail catheter with saline and advance it over the wire and through the WATCHMAN access sheath. Then, remove the guidewire and connect a syringe with contrast dye to the pigtail. 
11. While viewing TEE, turn the WATCHMAN access sheath counterclockwise to align the sheath more anterior and advance the pigtail in the desired position in the distal portion of the LAA.

12. Once the pigtail catheter has reached the LAA, obtain angiography at a right anterior oblique (RAO) of $20-30^{\circ}$, caudal $20-30^{\circ}$. Then perform TEE with a minimum of a $0-135^{\circ}$ sweep. This is most important when the sheath is advanced near the wall or apex of the LAA and while advancing more distally in any anatomy.

13. Carefully advance the access sheath over the pigtail catheter in the most superior lobe: in angiography this is the LAA-lobe situated around 2 o'clock at a right anterior oblique (RAO) of 20-30 ${ }^{\circ}$, caudal 20-30 ${ }^{\circ}$, in TEE it is the most right LAA-lobe at $135^{\circ}$.

14. Under fluoroscopic guidance, advance the WATCHMAN access sheath to the desired depth in the LAA based on the marker band corresponding to the appropriate size.

15. Once the pigtail is as distal as possible, inject the contrast dye.

16. Select the appropriate WATCHMAN device size based on maximum LAA ostium dimensions and sizing ${ }^{22}$.

17. Under sterile conditions, remove the WATCHMAN device from the packaging inspect it for damage. To confirm the device is attached to the core wire, open the hemostatic valve and retract the device approximately $1 \mathrm{~cm}$.

18. Align the distal tip of the device with the marker band. The WATCHMAN device should not protrude.

19. Attach a large $60 \mathrm{cc}$ syringe containing saline. Flush the system several times to remove any air. Then, submerge the tip of the delivery catheter in saline and tap it to remove bubbles.

20. Recheck the position of the access sheath by angiography. Loosen the access sheath valve and slowly remove the pigtail catheter from the sheath. Allow the sheath to back bleed.

21. To avoid introducing air, inject saline through the flush port so that it drips from the delivery catheter, then, introduce the WATCHMAN device system.

22. Under fluoroscopic guidance, slowly advance the delivery catheter into the access sheath until the most distal marker band on the access sheath lines up with the marker band on the delivery catheter and stabilize the delivery catheter.

23. Retract the access sheath and snap it onto the delivery catheter. Using fluoroscopy, reconfirm position of the delivery catheter tip. Do not advance the access sheath once the delivery system has been snapped into it.

24. If repositioning is required, remove the delivery system, reinsert the pigtail catheter and advance the access sheath into the proper position

25. Once the delivery system is positioned, loosen the valve on the WATCHMAN delivery catheter. Observe the distal end of device to ensure that no forward advancement or repositioning relative to ostium occurs.

26. Then, to deploy the device, hold the deployment knob stationary and slowly retract the access sheath/delivery catheter assembly with a slow stable motion over a period of 3-5 sec.

27. Withdraw the access sheath/delivery catheter assembly a few centimeters from the device to align it with the LAA, leaving the core wire attached.

28. Once the device has been deployed, inject contrast again. Then, using fluoroscopy and TEE, confirm the Position, Anchor, Size and Seal (PASS) device release criteria have been met as follows:

29. Position: Confirm the device is properly positioned by ensuring that the plane of maximum diameter of the device spans the LAA ostium and is at or just distal to the orifice of the LAA.

30. Anchor: Confirm the device is anchored in place, by withdrawing the access sheath/delivery catheter assembly $1-2 \mathrm{~cm}$ from the face of the device then gently retracting and releasing the deployment knob. The LAA and device should move in unison.

31. Size: Confirm that the device is appropriately sized by measuring the plane of the maximum diameter of the device using TEE in the standard 4 views $0^{\circ}, 45^{\circ}, 90^{\circ}$, and $135^{\circ}$, ensuring the threaded insert is visible. The device should be $80-92 \%$ of the original size.

32. Seal: using color Doppler, ensure that all of the lobes distal to device are sealed. Color flow should not be detected near the device. If there is a gap or jet around the device that is larger than $5 \mathrm{~mm}$, the device should be repositioned or fully recaptured and replaced.

33. If all release criteria are met, move the access sheath/delivery catheter up to the face of the device and rotate the deployment knob $3-5$ turns counterclockwise to release.

34. After release, perform angiography with contrast dye to document that the device is still in place. Then, using TEE, recheck the size and seal. Remove the sheath assembly from the left atrium.

35. If the device is too distal or proximal in the LAA or the device criteria are not met, it may be necessary to partially or fully recapture the device (for details please see the 2012 JoVE article by Möbius-Winkler and colleagues) ${ }^{22}$.

\section{Post-procedure}

1. Once proper placement of the device has been confirmed, remove the sheath. The femoral vein should then be compressed for a few hours and the patient should be carefully monitored for at least $6 \mathrm{hr}$ with blood pressure and heart rate monitoring.

2. Check for hematoma and/or bleeding at regular intervals according to institutional guidelines. The patient should be administered antibiotic prophylaxis per the American Heart Association's guidelines. NOTE: Post procedure heparin is not recommended.

3. Once the patient is awake, perform a neurological examination.

4. The patient should remain on warfarin and $81 \mathrm{mg}$ aspirin for a minimum of 45 days after the implant procedure (internal normalized ratio $=$ 2.0-3.0).

5. The patient should be hospitalized overnight and discharged the next day. A transthoracic echocardiogram (TTE) or chest x-ray may be performed to confirm the absence of pericardial effusion.

6. At 45 days, assess the WATCHMAN device placement using TEE. Cessation of warfarin is at physician discretion, if the LAA is closed completely and thrombus on the device was ruled out. If flow is noted around the device greater than $5 \mathrm{~mm}$, consideration should be given to keep the patient on warfarin until it has decreased to less than $5 \mathrm{~mm}$.

7. Patients ceasing warfarin should begin $75 \mathrm{mg}$ clopidogrel daily through 6 months post-implant and continue taking aspirin daily indefinitely.

8. Prescribe the appropriate endocarditis prophylaxis for 6 months following implantation. Continuing endocarditis prophylaxis beyond 6 months is at physician discretion. 


\section{Representative Results}

In a preliminary study of WATCHMAN implantation in 10 patients that had a high risk of stroke and bleeding, 9 of the patients received the device after a catheter ablation. The data from this study is published in the Netherlands Heart Journal, and is presented here with permission ${ }^{21}$. All patients included in the study were fully informed about the procedure and signed a consent form. The study was approved by the hospital's ethics committee and all procedures were performed in accordance with the hospital's ethics standards and the Helsinki Declaration of 1975 (revised in 2008).

The characteristics of these patients are summarized in Table 1. All patients had documented AF with a high stroke risk $\left(\mathrm{CHADS}_{2}>1\right)$ and all but one of the patients had history of stroke. LAA closure was indicated as an alternative to OAC for the following reasons: stroke during OAC therapy $(3 / 10)$, severe bleeding during OAC therapy $(2 / 10)$ and patient preference $(5 / 10)$. Prior to the procedure all patients were taking a vitamin $\mathrm{K}$ antagonist. The median $\mathrm{CHADS}_{2}$ score for this group was 3 , with a median $\mathrm{CHA}_{2} \mathrm{DS}_{2}$-VASc score of 3.5 and a median HAS-BLED score of 1.5. Prior to the procedure, all patients underwent TEE to exclude LAA thrombus and to rule out structural cardiac abnormalities. Among patients, the average maximum LAA diameter was $20.2 \pm 2.2 \mathrm{~mm}$ with an average depth of $27.9 \pm 5.4 \mathrm{~mm}$. $40 \%$ of the patients had a multi-lobular atrial appendage.

Table 2 outlines procedural characteristics. The WATCHMAN LAA closure device was successfully placed in all 10 patients. Based on patient anatomy determined during preliminary TEE, three different device sizes were used. The median total procedure time was 104 min (range 75-167 $\mathrm{min}$ ), including a median $56 \mathrm{~min}$ (range 38-137 $\mathrm{min}$ ) for LAA occlusion. During the procedure, an asymptomatic catheter thrombus occurred in one patient. The thrombus was aspirated and the patient received an extra bolus of heparin. Prolonged femoral vein bleeding in this patient necessitated a blood transfusion and extended hospitalization. All other patients were discharged from the hospital on the day following the procedure (Table 2). OAC was continued at least until TEE was performed at 45 days, at which point the device position and closure rate was assessed.

The findings at the 45-day patient follow-up are summarized Table 3. Successful device implantation was defined as long-term sealing of the LAA as measured by TEE 45 days after the procedure without major adverse events. Residual flow $(<5 \mathrm{~mm})$ around the device was allowed after the procedure (according to the PROTECT-AF study). Importantly, no thromboembolic cerebral events occurred. Minimal residual flow was observed in 3 patients. TEE revealed asymptomatic embolization of the device in one patient, and the device was percutaneously retrieved via the femoral vein. Thrombus was not seen on the device in any cases. One patient required a redo-ablation procedure 3 months after the initial procedure, in which pulmonary vein isolation was successful. The implanted LAA device was not affected by and did not interfere with the procedure. Three of the 10 patients were able to discontinue warfarin treatment at 45 days. In the PROTECT-AF ${ }^{18}$ and Continues Access Registry ${ }^{29}$ device embolization occurred in 3 patients out of the 1,002 patients implanted.

Figure 2 shows a TEE screen capture of the LAA in one example patient in which no residual flow is observed 45 days post-procedure. In contrast, flow around the device, as evidenced by color Doppler, is seen for another patient (Figure 3).

\begin{tabular}{|c|c|}
\hline \multicolumn{2}{|l|}{ Baseline Characteristics } \\
\hline Number, $\mathbf{n}$ & 10 \\
\hline Age (years) & $61.6+/ 9.6$ \\
\hline Sex (\%female) & $5(50)$ \\
\hline Median $\mathrm{CHADS}_{2}$ & $3(2-4)$ \\
\hline 2 & $4(40)$ \\
\hline 3 & $4(40)$ \\
\hline 4 & $2(20)$ \\
\hline $\mathrm{CHA}_{2} \mathrm{DS}_{2}$-VASc & $3.5(2-6)$ \\
\hline HAS-BLED & $1.5(1-4)$ \\
\hline Vitamin $\mathrm{K}$ antagonist, $\mathrm{n}(\%)$ & $10(100)$ \\
\hline \multicolumn{2}{|l|}{ Indication, n(\%) } \\
\hline Bleeding with OAC & $2(20)$ \\
\hline Stroke using OAC & $3(30)$ \\
\hline Patient Preference & $5(50)$ \\
\hline \multicolumn{2}{|l|}{ LAA, mm } \\
\hline LAA width & $20.2+/ 2.2$ \\
\hline LAA length & $27.9+/-5.4$ \\
\hline Multilobular & $4(40)$ \\
\hline
\end{tabular}


Table 1. Baseline characteristics of patients enrolled in the study. Data reprinted with permission from the Netherlands Heart Journal ${ }^{21}$. All data are presented as mean $+/$ - standard deviation, as number with percentage $(n(\%))$ or median with lower and upper range, $\mathrm{N}=\mathrm{number}$, $\mathrm{mm}=$ millimeters, $\mathrm{OAC}=$ oral anticoagulation, $\mathrm{LAA}=$ left atrial appendage.

\begin{tabular}{|c|c|}
\hline \multicolumn{2}{|l|}{ Procedural characteristics } \\
\hline PVAC, $n(\%)$ & $9(90)$ \\
\hline Size device $(\mathrm{mm})$ & $24(21-27)$ \\
\hline 21 & $1(10)$ \\
\hline 24 & $8(80)$ \\
\hline 27 & $1(10)$ \\
\hline LAA Occlusion Time (min) & $56(38-137)$ \\
\hline Total procedural time (min) & $104(75-167)$ \\
\hline Devices, $\mathrm{n}$ & $2(1-3)$ \\
\hline \multicolumn{2}{|l|}{ Complications, $n(\%)$} \\
\hline Catheter thrombus & $1(10)^{*}$ \\
\hline Inguinal bleeding & $1(10)^{*}$ \\
\hline Pericardial effusion & $0(0)^{*}$ \\
\hline \multicolumn{2}{|l|}{ TEE } \\
\hline Successful implantation & $10(100)$ \\
\hline Residual Flow & $1(10)$ \\
\hline Hospitalization, days & $2(2-7)$ \\
\hline
\end{tabular}

Table 2. Procedural characteristics. Data reprinted with permission from the Netherlands Heart Journal ${ }^{21}$. All data are presented as mean $+/-$ standard deviation, as number with percentage $(n(\%))$ or median with lower and upper range, $N=$ number, mm=millimeters, PVAC=pulmonary vein ablation catheter, TEE=Transesophageal echocardiography. *Both in the same patient.

\begin{tabular}{|l|l|}
\hline \multicolumn{2}{|l|}{ Follow-up characteristics at $\mathbf{4 5}$ days } \\
\hline Number, $\mathrm{n}(\%)$ & 10 \\
\hline TEE, $\mathrm{n}(\%)$ & $3(33)$ \\
\hline Residual flow & $1(10)$ \\
\hline Device embolization & $0(0)$ \\
\hline Thrombus on device & $6(67)$ \\
\hline Vitamin K antagonist, $\mathrm{n}(\%)$ & \multicolumn{2}{|l|}{} \\
\hline Complications during follow-up, $\mathrm{n}(\%)$ & $0(0)$ \\
\hline Death & $0(0)$ \\
\hline Stroke or TIA & $1(10)$ \\
\hline Bleeding & \\
\hline
\end{tabular}

Table 3. 45-day follow-up. Data reprinted with permission from Netherlands Heart Journal ${ }^{21}$. All data are presented as mean $+/-$ standard deviation, as number with percentage $(\mathrm{n}(\%))$ or median with lower and upper range, $\mathrm{N}=$ number, $\mathrm{mm}=$ millimeters, TEE=Transesophageal echocardiography, $\mathrm{TIA}=$ transient ischemic attack. 


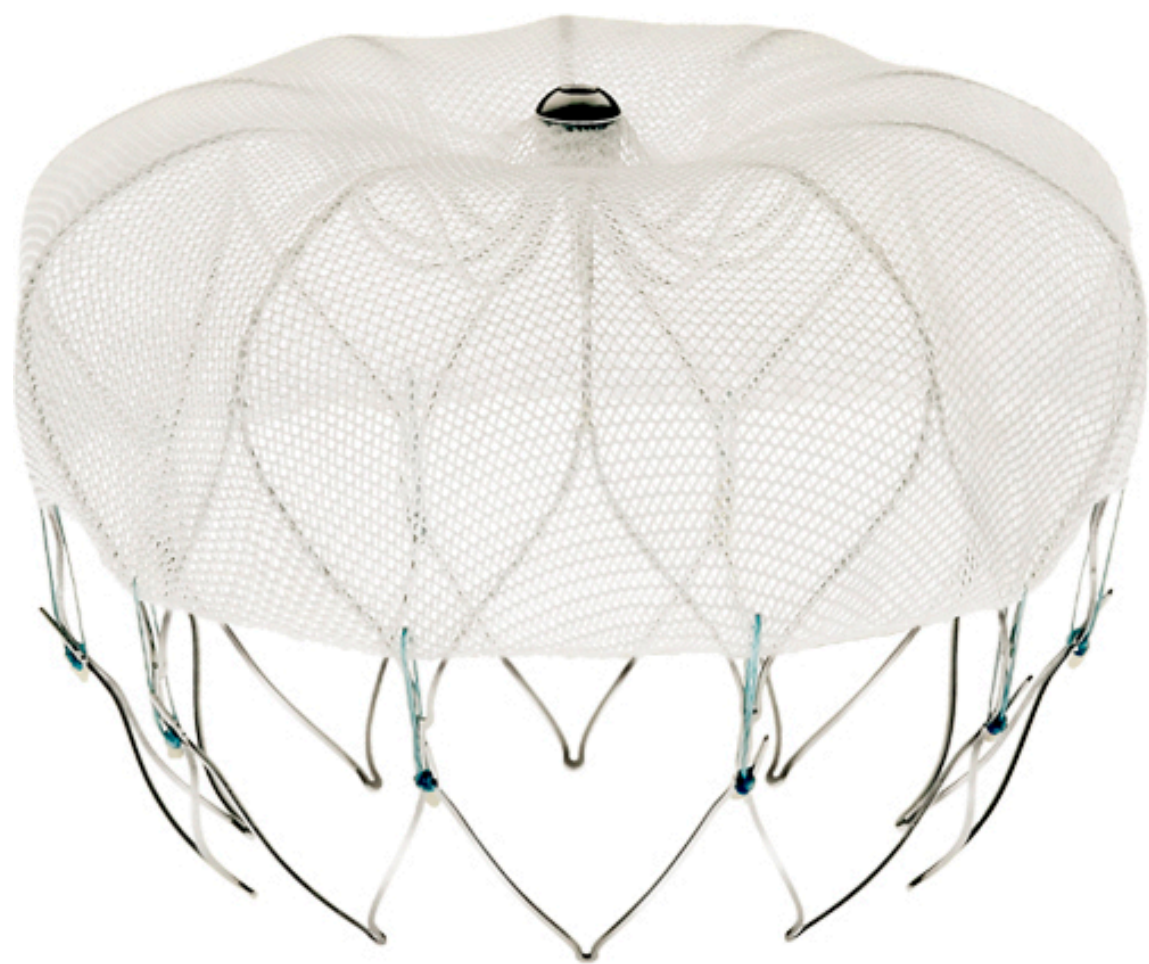

Figure 1. The WATCHMAN Device. The WATCHMAN device is a nitinol cage covered with a Polyethyl terephthalate membrane. Fixation barbs around the perimeter secure the device in the LAA.

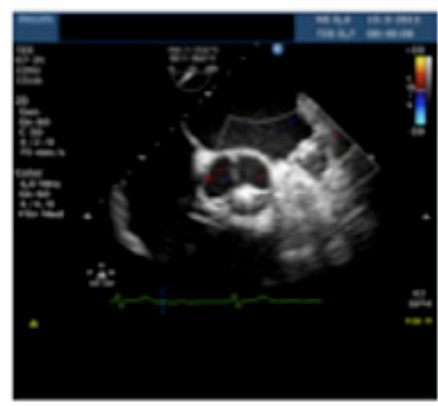

$45^{\circ}$

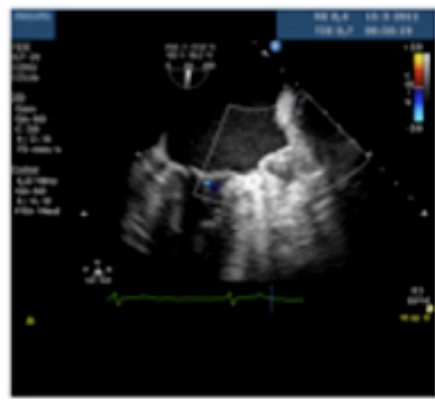

$90^{\circ}$

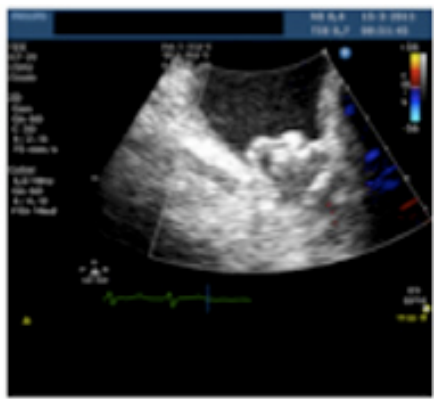

$135^{\circ}$

Figure 2. A complete LAA isolation or occlusion. TEE with color Doppler demonstrating proper positioning and occlusion at 45 days postprocedure from different TEE-angulations in a single patient. As the TEE probe looks at the device between $0-180^{\circ}$, there is no flow in the appendage and that the device is appropriately placed in all views.

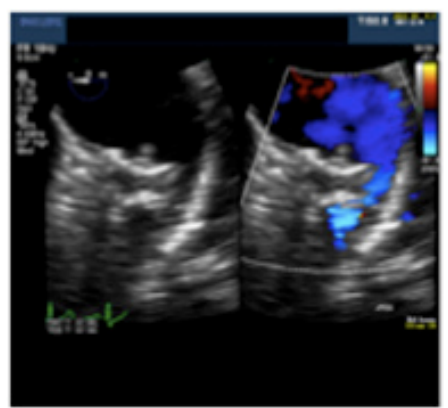

$0^{\circ}$

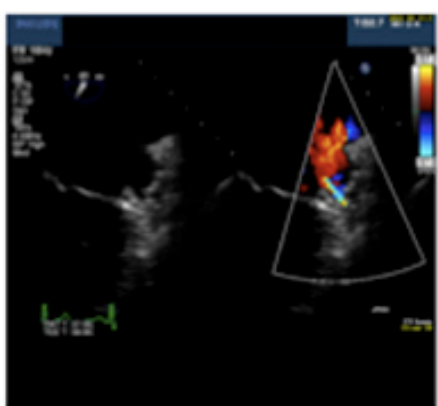

$60^{\circ}$

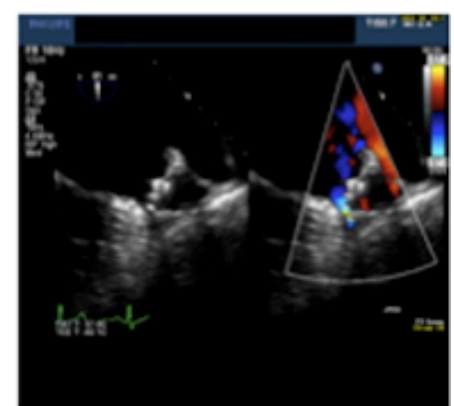

$90^{\circ}$

Figure 3. An incomplete LAA occlusion. TEE with color Doppler 45 days post-procedure demonstrating flow (leak) around the device. As the TEE probe looks at the device between $0-180^{\circ}$, a small gap $(<5 \mathrm{~mm})$ with color flow is seen around the device. These findings still can be qualified as successful sealing. Therefore OAC can be stopped. All images are from the same patient. 
Discussion

The preceding text describes a procedure for combined catheter ablation and left atrial appendage occlusion as treatment for AF and prevention of associated thromboembolic events. Preliminary data indicate that this procedure can be safely performed in patients at high risk for stroke. No technical difficulties have been encountered in performing the combined procedure. While no improvement to either procedure can be expected since they do not influence each other, the combined procedure may offer several advantages over performing the procedures separately. The combined procedure enables simultaneous treatment of AF symptoms and reduction of stroke risk, improving quality of life for those affected by AF. It may also present drug refractory-patients a treatment option by allowing them to undergo implantation of the WATCHMAN device without subsequent warfarin treatment. Furthermore, the need for, and associated risk of, a repeated left atrial intervention and transseptal puncture is reduced should LAA closure become desirable during follow-up.

While CA is an established treatment for symptoms of AF, recent data suggest that it is also effective in reducing stroke. In a retrospective analysis of matched AF patient pairs, Reynolds and colleagues saw a significant decrease in the relative risk of stroke and transient ischemic attack events among patients who underwent ablation compared with those undergoing antiarrhythmic drug therapy ${ }^{16}$. Data also indicates that amputation of the LAA or occlusion via surgical ligation or device placement effectively reduces embolic events. These methods appear promising: in a study of patients undergoing mitral valve replacement those that underwent surgical ligation had significantly fewer strokes (3 vs. $17 \%)^{19,23}$. A less invasive, thoracoscopic ligation using an loop snare procedure has been performed successfully in 14 of 15 patients with stroke occurring in 2 patients (4\%) over the following 42 months ${ }^{19,24}$.

Several percutaneous catheter LAA occlusion devices have been developed including the Amplatzer Cardiac Plug (AGA medical), the Transcatheter Patch (Custom Medical Devices), the percutaneous LAA Transcatheter occlusion (PLAATO) system, and the WATCHMAN LAA occlusion device. At present, clinical studies regarding safety and efficacy are published only for PLAATO (which is no longer available) and the WATCHMAN. The PLAATO demonstrated safety and efficacy and offers proof of concept for LAA closure using a nitinol filter-based device. The device was a self-expanding nitinol cage covered in polytetrafluoroethylene that prevented blood from entering the LAA ${ }^{25}$. A non-randomized study of 64 patients with a CHADS ${ }_{2}$ of $>2$ demonstrated complete occlusion in more than $98 \%$ of patients. After 5 years, the stroke/transient ischemic attack rate was reduced to $3.8 \%$ compared to the expected $6.6 \%$ in a population ${ }^{26}$

The data obtained from the PROTECT AF study ${ }^{18}$, which compared the efficacy of the WATCHMAN device to treatment with warfarin further support a device-based approach of LAA occlusion. This randomized study showed that implantation WATCHMAN was found to be non-inferior in terms of all-cause stroke and all-cause mortality in OAC eligible patients, raising the possibility that the procedure could eventually be used as an alternative to chronic warfarin therapy in patients who are drug-refractive ${ }^{18}$. In the study published in Netherlands Heart Journal in $2012^{21}$, we successfully implanted the device in 10 patients, 9 of whom underwent concurrent CA. Note that although residual flow was seen in 3 cases at 45 days follow up, Viles-Gonzalez and colleagues have shown that this residual flow around the device is not associated with an increased risk of thromboembolic events ${ }^{27}$

While the available data support both CA and LAA occlusion using the WATCHMAN device as effective treatments for AF, both procedures are associated with complications. A worldwide survey of CA in 2010 showed that complications occur in $4.5 \%$ of patients ${ }^{14}$. The most significant complications include: death, cerebral thromboembolic events, extrapericardial pulmonary vein perforation, tamponade, stenosis, and atypical flutter of new onset (iatrogenic) ${ }^{14,15,28}$. Procedural complications that may result from WATCHMAN device placement procedure include serious pericardial effusion, device embolization, procedure-related stroke ${ }^{18}$. In both cases, the incidence of complications has been found to depend on operator experience ${ }^{15,29}$.

In the 2012 study of the combined procedure, complications were limited to one device embolism. Half of the patients did not have documented recurrence of $\mathrm{AF}$ at 3-month follow-up. The other half of the patients required at least one direct current cardioversion. One patient underwent repeat ablation and it was successful ${ }^{21}$. Though this study was of a small population and does not provide a direct comparison with other methods of treatment, studies of safety in each of the independent procedures ${ }^{29,30}$ suggest that the potential benefits of treatment offsets the increase in safety risk. Additional safety issues to consider are the longer procedural times, requirement for general anesthesia, and the increased risk of GI-tract bleeding with TEE monitoring, which must accompany WATCHMAN placement.

While the procedure described herein describes a combined procedure of WATCHMAN placement and PV CA, it is important to note that WATCHMAN placement does not preclude a strategy of electrical isolation of the LAA. A recent study by Di Biase and colleagues demonstrated that in a selected patient group with recurrent AF, $27 \%$ showed foci arising from the LAA after an initial ablation, which was optimally treated via complete circumferential LAA isolation ${ }^{31}$. This patient group might especially benefit from LAA closure because electrical isolation of the LAA could lead to LAA stasis even during sinus rhythm, making it more thrombogenic. In patients for whom AF ablation and LAA closure is considered, one could first perform an electrophysiological study with pharmacological challenges to detect and ablate possible LAA triggers before implantation of the WATCHMAN device ${ }^{31}$.

Given the present study is not randomized, and is limited to a 45-day follow up with 10 high-risk patients, numerous questions remain to be addressed: Is the combined CA/WATCHMAN implantation procedure more effective in stroke prevention and relief of AF symptoms than either method alone? How do the benefits of the procedure compare if the order of the procedures is reversed, with LAA occlusion first? How does the safety of the procedure compare to either device implantation or CA alone? Is this effective in lower-risk patients? Is the need for future ablations affected by the combined procedure? Can the procedure be performed with equal success in patients not taking warfarin? Future studies will include a randomized control study to examine whether combining these techniques improves patient outcome and quality of life in these patients.

Note that when performing this procedure it is important to carefully evaluate the LAA anatomy when selecting the device size and to thoroughly check the placement of the device before releasing it. It is also very important to ensure that the depth of the landing zone for the device is equal or greater than the maximum width of the LAA ostium. These steps are critical for patient safety and effectiveness of the procedure. 


\section{Disclosures}

The Cardiology Department of St. Antonius Hospital (Nieuwegein, the Netherlands) receives proctoring fees for training/educational services from Atritech/Boston Scientific.

Production and Free Access to this article is sponsored by Boston Scientific.

\section{Acknowledgements}

The authors would like to thank Ted van de Beek of Boston Scientific for his support during the preparation of this manuscript.

\section{References}

1. Wolowacz, S.E., Samuel, M., Brennan, V.K., Jasso-Mosqueda, J.G., \& Van Gelder, I.C. The cost of illness of atrial fibrillation: a systematic review of the recent literature. Europace : European pacing, arrhythmias, and cardiac electrophysiology. Journal of the Working groups on cardiac pacing, arrhythmias, and cardiac cellular electrophysiology of the European Society of Cardiology. 13, 1375-1385 (2011).

2. Miyasaka, Y., et al. Secular trends in incidence of atrial fibrillation in Olmsted County, Minnesota, 1980 to 2000, and implications on the projections for future prevalence. Circulation. 114, 119-125 (2006).

3. Psaty, B.M., et al. Incidence of and risk factors for atrial fibrillation in older adults. Circulation. 96, 2455-2461 (1997)

4. Ames, A. \& Stevenson, W.G. Cardiology patient page. Catheter ablation of atrial fibrillation. Circulation. 113, e666-668 (2006).

5. Bin Salih, S.A., et al. Clinical characteristics of patients with atrial fibrillation at a tertiary care hospital in the central region of Saudi Arabia. J. Family Community Med. 18, 80-84 (2011).

6. Lloyd-Jones, D.M., et al. Lifetime risk for development of atrial fibrillation: the Framingham Heart Study. Circulation. 110, 1042-1046 (2004).

7. Hart, R.G., Pearce, L.A., \& Aguilar, M.I. Meta-analysis: antithrombotic therapy to prevent stroke in patients who have nonvalvular atrial fibrillation. Ann. Intern. Med. 146, 857-867 (2007).

8. Nieuwlaat, R., et al. Atrial fibrillation management: a prospective survey in ESC member countries: the Euro Heart Survey on Atrial Fibrillation. European Heart Journal. 26, 2422-2434 (2005).

9. Gage, B.F., et al. Adverse outcomes and predictors of underuse of antithrombotic therapy in medicare beneficiaries with chronic atria fibrillation. Stroke: A Journal of Cerebral Circulation. 31, 822-827 (2000).

10. Hylek, E.M., Evans-Molina, C., Shea, C., Henault, L.E., \& Regan, S. Major hemorrhage and tolerability of warfarin in the first year of therapy among elderly patients with atrial fibrillation. Circulation. 115, 2689-2696 (2007).

11. Sudlow, M., Thomson, R., Thwaites, B., Rodgers, H., \& Kenny, R.A. Prevalence of atrial fibrillation and eligibility for anticoagulants in the community. Lancet. 352, 1167-1171 (1998).

12. Schweizer, P.A., Becker, R., Katus, H.A., \& Thomas, D. Dronedarone: current evidence for its safety and efficacy in the management of atrial fibrillation. Drug Des. Devel. Ther. 5, 27-39 (2011).

13. Echt, D.S., et al. Mortality and morbidity in patients receiving encainide, flecainide, or placebo. The Cardiac Arrhythmia Suppression Trial. The New England Journal of Medicine. 324, 781-788 (1991).

14. Cappato, R., et al. Updated worldwide survey on the methods, efficacy, and safety of catheter ablation for human atrial fibrillation. Circulation. Arrhythmia and Electrophysiology. 3, 32-38 (2009).

15. Kumagai, K. Catheter ablation of atrial fibrillation. Circ. J. 75, 2305-2311 (2011).

16. Reynolds, M.R., et al. Health outcomes with catheter ablation or antiarrhythmic drug therapy in atrial fibrillation: results of a propensitymatched analysis. Circulation. Cardiovascular Quality and Outcomes. 5, 171-181 (2012).

17. Blackshear, J.L. \& Odell, J.A. Appendage obliteration to reduce stroke in cardiac surgical patients with atrial fibrillation. The Annals of Thoracic Surgery. 61, 755-759 (1996)

18. Holmes, D.R., et al. Percutaneous closure of the left atrial appendage versus warfarin therapy for prevention of stroke in patients with atrial fibrillation: a randomised non-inferiority trial. Lancet. 374, 534-542 (2009).

19. Singh, I.M. \& Holmes, D.R., Jr. Left atrial appendage closure. Current Cardiology Reports. 12, 413-421 (2010).

20. Velthuis, S., et al. Left Atrial Appendage Closure for Stroke Prevention in Patients with Atrial Fibrillation and Hereditary Hemorrhagic Telangiectasia. Case Reports in Cardiology. 2012, 3 (2012).

21. Swaans, M.J., Post, M.C., Rensing, B.J., \& Boersma, L.V. Percutaneous left atrial appendage closure for stroke prevention in atrial fibrillation. Netherlands Heart Journal: Monthly Journal of the Netherlands Society of Cardiology and the Netherlands Heart Foundation. 20, 161-166 (2012).

22. Mobius-Winkler, S., et al. The WATCHMAN left atrial appendage closure device for atrial fibrillation. J. Vis. Exp. (60), e3671, doi:10.3791/3671 (2012).

23. Garcia-Fernandez, M.A., et al. Role of left atrial appendage obliteration in stroke reduction in patients with mitral valve prosthesis: a transesophageal echocardiographic study. Journal of the American College of Cardiology. 42, 1253-1258 (2003).

24. Blackshear, J.L., et al. Thoracoscopic extracardiac obliteration of the left atrial appendage for stroke risk reduction in atrial fibrillation. Journal of the American College of Cardiology. 42, 1249-1252 (2003).

25. Block, P.C. Watching the watchman. Journal of the American College of Cardiology. 49, 1496-1497 (2007).

26. Block, P.C., et al. Percutaneous left atrial appendage occlusion for patients in atrial fibrillation suboptimal for warfarin therapy: 5 -year results of the PLAATO (Percutaneous Left Atrial Appendage Transcatheter Occlusion) Study. JACC. Cardiovascular interventions. 2, 594-600 (2009).

27. Viles-Gonzalez, J.F., et al. Incomplete occlusion of the left atrial appendage with the percutaneous left atrial appendage transcatheter occlusion device is not associated with increased risk of stroke. Journal of Interventional Cardiac Electrophysiology: An International Journal of Arrhythmias and Pacing. 33, 69-75 (2012). 
28. Cappato, R., et al. Worldwide survey on the methods, efficacy, and safety of catheter ablation for human atrial fibrillation. Circulation. 111, 1100-1105 (2005).

29. Reddy, V.Y., Holmes, D., Doshi, S.K., Neuzil, P., \& Kar, S. Safety of percutaneous left atrial appendage closure: results from the Watchman Left Atrial Appendage System for Embolic Protection in Patients with AF (PROTECT AF) clinical trial and the Continued Access Registry. Circulation. 123, 417-424 (2011).

30. Oral, H., et al. Pulmonary vein isolation for paroxysmal and persistent atrial fibrillation. Circulation. 105, 1077-1081 (2002).

31. Di Biase, L., et al. Left atrial appendage: an underrecognized trigger site of atrial fibrillation. Circulation. 122, 109-118 (2010). 